\title{
SHORT PROOF OF TWO CASES OF CHVÁTAL'S CONJECTURE
}

\author{
JORGE OLARTE, FRANCISCO SANTOS, AND JONATHAN SPREER
}

\begin{abstract}
In 1974 Chvátal conjectured that no intersecting family $\mathcal{F}$ in a downset can be larger than the largest star. In the same year Kleitman and Magnanti proved the conjecture when $\mathcal{F}$ is contained in the union of two stars, and Sterboul when $\operatorname{rank}(\mathcal{F}) \leq 3$. We give short self-contained proofs of these two statements.
\end{abstract}

\section{INTRODUCTION}

A downset, hereditary set, independence system or (abstract) simplicial complex $\mathcal{C}$ is a family of subsets of some finite ground set closed under taking subsets. Using nomenclature from simplicial complexes we call faces the elements of $\mathcal{C}$ and vertices, edges and triangles, respectively, the faces of sizes 1, 2 and 3. The star of a vertex $a$, written $\operatorname{st}_{\mathcal{C}}(a)$, is the family of all faces containing $a$. It is an example of an intersecting family in $\mathcal{C}$, that is, a set of faces that pairwise intersect.

Chvátal's 45-year-old conjecture, inspired by the classical result of Erdôs, Ko and Rado [3] for the complete, uniform complex $\left(\begin{array}{c}{[n]} \\ \leq k\end{array}\right)$, states that stars always achieve the maximal cardinality among intersecting families in $\mathcal{C}$ :

Conjecture 1.1 (Chvátal [1]). Let $\mathcal{F}$ be an intersecting family in a simplicial complex $\mathcal{C}$. Then, there exists a vertex $a$ in $\mathcal{C}$ such that $|\mathcal{F}| \leq\left|\operatorname{st}_{\mathcal{C}}(a)\right|$.

Note that there is no loss of generality in assuming that $\mathcal{C}$ is the smallest downset containing $\mathcal{F}$ (in other words, $\mathcal{C}$ is generated by $\mathcal{F}$ ). Throughout the article we assume this and give short proofs of the following old and very recent known cases.

Theorem 1.2 (Kleitman and Magnanti [4, Theorem 2]). Let $\mathcal{F}$ be an intersecting family contained in the union of two stars, $\mathcal{F} \subset \operatorname{st}(a) \cup \operatorname{st}(b)$. Then $|\mathcal{F}| \leq$ $\max (|\operatorname{st}(a)|,|\operatorname{st}(b)|)$.

Theorem 1.3 (Sterboul [6, Theorem 2]). Chvátal's conjecture holds if all elements of $\mathcal{F}$ have size three or less.

Equivalently, this result settles Chvátal's conjecture for rank at most three. It was recently reproven by Czabarka, Hurlbert and Kamat [2, Theorem 1.4]. We thank G. Hurlbert for pointing us towards reference [6].

Our proofs are inspired by our recent work with Stump on a related EKR problem [5].

(J. Olarte and J. Spreer) Institut fÜr Mathematik, Freie Universität Berlin, Germany

(F. Santos) Department of Mathematics, Statistics and Computer Science, UniverSity of Cantabria, Spain

E-mail addresses: olarte@zedat.fu-berlin.de, jonathan.spreer@fu-berlin.de, francisco.santos@unican.es.

Date: June 6, 2021.

2010 Mathematics Subject Classification. Primary 05E45; Secondary 52C10, 05 D05.

Key words and phrases. Erdôs-Ko-Rado property, Chvátal's Conjecture.

The three authors are supported by the Einstein Foundation, Berlin. F. Santos is also supported by grant MTM2014-54207-P of the Spanish Ministry of Science. 


\section{INTERSECTING FAMILIES CONTAINED IN TWO STARS}

Lemma 2.1. Let $\mathcal{F}$ be an intersecting family in $\mathcal{C}$. Let $a, b, v$ be three vertices of $\mathcal{C}$ and assume that every $B \in \mathcal{F}$ with $v \in B$ intersects $\{a, b\}$. Define

$$
\begin{aligned}
R_{a}(v) & :=\{B \in \mathcal{F}: a, v \in B, b \notin B, B \backslash v \notin \mathcal{F}\}, \\
R_{b}(v) & :=\{B \in \mathcal{F}: b, v \in B, a \notin B, B \backslash v \notin \mathcal{F}\} .
\end{aligned}
$$

Then, $\mathcal{F}^{\prime}:=\mathcal{F} \backslash R_{b}(v) \cup\left\{B \backslash v: B \in R_{a}(v)\right\}$ is also an intersecting family.

Proof. All sets in $\left\{B \backslash v: B \in R_{a}(v)\right\}$ intersect one another since they all contain $a$. We thus only need to show that every $B_{1} \in \mathcal{F} \backslash R_{b}(v)$ intersects every $B_{2} \in R_{a}(v)$ in an element different from $v$. If $v \notin B_{1}$ this is obvious since $B_{1}$ and $B_{2}$ are both in $\mathcal{F}$ and thus they meet. If $a \in B_{1}$ this is obvious too, since then $a \in B_{1} \cap B_{2}$. Hence, assume $B_{1}$ contains $v$ but not $a$. Our hypotheses imply that $b \in B_{1}$ and since $B_{1} \notin R_{b}(v)$ we have that $B_{1} \backslash v \in \mathcal{F}$. Thus, $\left(B_{1} \backslash v\right) \cap B_{2}$ indeed meet.

Theorem 1.2 is a direct consequence of the following statement.

Corollary 2.2. Let $\mathcal{F} \subset \mathcal{C}$ be an intersecting family such that $\mathcal{F} \subset \operatorname{st}(a) \cup \operatorname{st}(b)$ and neither $\operatorname{st}(a)$ nor $\operatorname{st}(b)$ contains $\mathcal{F}$. Then there exists an intersecting family $\mathcal{F}^{\prime} \subset \mathcal{C}$, $\mathcal{F}^{\prime} \subset \operatorname{st}(a) \cup \operatorname{st}(b)$, such that either $\left|\mathcal{F}^{\prime}\right|>|\mathcal{F}|$ or $\left|\mathcal{F}^{\prime}\right|=|\mathcal{F}|$ but then $\mathcal{F}^{\prime}$ has smaller average size of elements than $\mathcal{F}$.

Proof. We first claim that there exists a vertex $v$ in $\mathcal{C}$ such that (at least) one of the sets $R_{a}(v)$ and $R_{b}(v)$ of the previous lemma is not empty. For this, let $B$ be a minimal face in $\mathcal{F}$ containing $a$ but not $b$ (it exists, or else the condition $\mathcal{F} \subset \operatorname{st}(a) \cup \operatorname{st}(b)$ implies $\mathcal{F} \subset \operatorname{st}(b))$. If $B=\{a\}$ then $\mathcal{F} \subset \operatorname{st}(a)$. If $B \neq\{a\}$ then for each $v \in B \backslash a$ we have $R_{a}(v) \neq \emptyset$.

Assume that either $\left|R_{a}(v)\right|>\left|R_{b}(v)\right|$ or $\left|R_{a}(v)\right|=\left|R_{b}(v)\right|$ and $\left|R_{a}(v)\right|$ has average size of sets smaller or equal than $\left|R_{b}(v)\right|$. This is no loss of generality since $\left|R_{a}(v)\right| \leq$ $\left|R_{b}(v)\right|$ implies $R_{b}(v)$ is not empty and we can exchange the roles of $a$ and $b$.

Hence, we have $\left|\mathcal{F}^{\prime}\right|=|\mathcal{F}|-\left|R_{b}(v)\right|+\left|R_{a}(v)\right| \geq|\mathcal{F}|$ with equality only if $\left|R_{a}(v)\right|=$ $\left|R_{b}(v)\right|$. In this case, since $\left|R_{a}(v)\right|$ has average size of sets smaller or equal than $\left|R_{b}(v)\right|$ and we substitute the sets of $R_{b}(v)$ with sets of size smaller than those of $R_{a}(v)$, the average size of sets in $\mathcal{F}^{\prime}$ is smaller than in $\mathcal{F}$.

\section{INTERSECTING FAMILIES OF RANK THREE}

To simplify notation, in what follows we omit braces when referring to a subset of the ground set and write, e. g., $a b c$ instead of $\{a, b, c\}$. In part (1) of the following statement, given a triangle $a b c \in \mathcal{F}$ we say that a second triangle $\tau \in \mathcal{F}$ is dangling from $a b c$ at one of the vertices $x \in a b c$ if $\tau \cap a b c=x$.

Lemma 3.1. Let $\mathcal{F}$ be an intersecting family consisting only of triangles. If any of the following conditions is satisfied, then there exists an intesecting family of size at least $|\mathcal{F}|$ containing an edge or vertex:

(1) Some triangle in $\mathcal{F}$ has one or no triangles dangling at some vertex;

(2) No two triangles in $\mathcal{F}$ share an edge;

(3) The graph of the complex generated by $\mathcal{F}$ is not complete.

Proof. Throughout the proof, let $a b c$ be a triangle in $\mathcal{F}$.

For part (1), if for some vertex, say $a$, there is only one triangle $\tau \in \mathcal{F}$ dangling at $a$, let $\mathcal{F}^{\prime}=\mathcal{F} \backslash\{\tau\} \cup\{b c\}$. If there is none, just add $b c$ to $\mathcal{F}$. 
For part (2), assume without loss of generality that among the triangles of $\mathcal{F}$ there are at least as many containing $a$ than $b$ or $c$. Let $\mathcal{F}^{\prime}$ consist of the triangle $a b c$ plus all other triangles $a x y \in \mathcal{F}$ together with their edges $a x$ and $a y$. Then $\mathcal{F}^{\prime} \subsetneq \operatorname{st}_{\mathcal{C}}(a)$ and $\left|\mathcal{F}^{\prime}\right| \geq|\mathcal{F}|$ since all edges $a x$ and ay are distinct.

For part (3), let $c$ and $v$ be vertices not spanning an edge. Let $a b c \in \mathcal{F}$ be a triangle containing $c$, and let $S_{v}=\{x \in a b c: \exists y$ with $v x y \in \mathcal{F}\}$. By the hypothesis, $S_{v} \subset a b$. The assumption that $\mathcal{C}$ is generated by the faces of $\mathcal{F}$ implies that $S_{v} \neq \emptyset$, so we assume $a \subset S_{v}$. If $S_{v}=a$ then we add $a y$ to $\mathcal{F}$ for each avy $\in \mathcal{F}$. Hence, assume for the rest that $S_{v}=a b$. Note that every element of $\mathcal{F}$ containing $v$ must contain either $a$ or $b$ since $\mathcal{F}$ is intersecting. In particular, we can apply Lemma 2.1. If one of $R_{a}(v)$ and $R_{b}(v)$ is non-empty this yields an intersecting family $\mathcal{F}^{\prime},\left|\mathcal{F}^{\prime}\right| \geq|\mathcal{F}|$, containing edges. If both $R_{a}(v)$ and $R_{b}(v)$ are empty, the only element of $\mathcal{F}$ containing $v$ is $a b v$ and we can add $a b$ to $\mathcal{F}$.

Proof of Theorem 1.3. Chvátal's conjecture holds when $\mathcal{F}$ contains a vertex (trivial) or an edge (Theorem 1.2; note that if $a b \in \mathcal{F}$, then trivially $\mathcal{F} \subset \operatorname{st}(a) \cup \operatorname{st}(b)$ ). Thus, we can assume that $\mathcal{F}$ consists entirely of triangles and, by Lemma 3.1, that it does not satisfy any of the three conditions listed in that lemma.

In particular, by part $(2)$ of the lemma, $\mathcal{F}$ contains two triangles $a b c$ and $a b x$ sharing an edge. Observe that all triangles dangling from $a b c$ at $c$ must contain $x$ since a triangle dangling at $c$ and not containing $x$ does not intersect $a b x$. Moreover:

- There are exactly two such triangles, say cxy and cxz. There are at least two by part (1) of Lemma 3.1. If there is a third triangle $c x v$ dangling at $c$, then every triangle in $\mathcal{F}$ must intersect $c x$ (otherwise it must contain $v, y$ and $z$ and intersect $a b c$, a contradiction). Hence, we can add $c x$ to $\mathcal{F}$ and apply Theorem 1.2.

- The only vertices of $\mathcal{C}$ are $a, b, c, x, y, z$. Assume there exists another vertex $v \in \mathcal{C}$. By part (3) of Lemma 3.1 the edge $c v$ is contained in some triangle $\tau \in \mathcal{F}$. By the previous item, $\tau$ is not dangling from $a b c$ at $c$ so without loss of generality $\tau=a c v$. Now, every triangle $\sigma \in \mathcal{F}$ dangling at $b$ must contain both $v$ and either $x$ or both $y$ and $z$. Since the latter is impossible, $b v x$ is the only possible triangle dangling at $b$, contradicting part (1) of Lemma 3.1.

Once we know there are exactly six vertices, observe that at most half of the $\left(\begin{array}{l}6 \\ 3\end{array}\right)=20$ triangles on six vertices, one from each complementary pair, can be in $\mathcal{F}$, so $|\mathcal{F}| \leq 10$. But the above implies that $\operatorname{st}_{\mathcal{C}}(c)$ contains at least the following 10 faces: the three triangles $a b c, c x y, c x z$ plus at least another triangle dangling from $c x y$ at $c$, the five edges $c a, c b, c x, c y, c z$, and $c$ itself.

\section{REFERENCES}

[1] V. Chvátal. Intersecting families of edges in hypergraphs having the hereditary property. In Hypergraph Seminar: Ohio State University 1972, vol. 411 of Lect. Notes Math., pp. 61-66. Springer Berlin Heidelberg, 1974.

[2] E. Czabarka, G. Hurlbert, and V. Kamat. Chvátal's conjecture for downsets of small rank, 2017. Preprint, arXiv:1703.00494.

[3] P. Erdôs, C. Ko, and R. Rado. Intersection theorems for systems of finite sets. Quart. J. Math. Oxford Ser. (2), 12:313-320, 1961.

[4] D. J. Kleitman and T. L. Magnanti. On the number of latent subsets of intersecting collections. J. Combinatorial Theory Ser. A, 16:215-220, 1974.

[5] Jorge A. Olarte, Francisco Santos, Jonathan Spreer, and Christian Stump. The ekr property for flag pure simplicial complexes without boundary, 2017. Preprint, arXiv:1710.02518.

[6] F. Sterboul. Sur une conjecture de V. Chvátal. In Hypergraph Seminar: Ohio State University 1972, vol. 411 of Lect. Notes Math., pp. 152-164. Springer Berlin Heidelberg, 1974. 